Pathologe 2010 · [Suppl 2] 31:328 DOI 10.1007/s00292-010-1309-y

Online publiziert: 13. August 2010

(c) Springer-Verlag 2010

\section{T. Yoshino}

Department of Pathology, Graduate School of Medicine,

Dentistry and Pharmaceutical Sciences, Okayama University, Okayama, Japan

\section{Katsuo Ogawa}

24.01.1920 - 15.10.2009

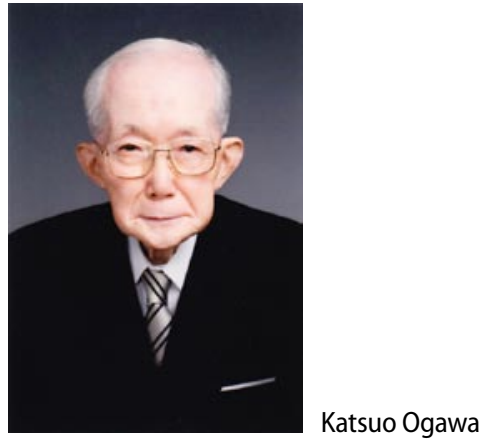

Prof. em. Dr. med. Katsuo Ogawa, ehemaliger Direktor des II. Pathologischen Institutes der Universität Okayama, Ehrenmitglied der Japanischen Gesellschaft fur Pathologie und korrespondierendes japanisches Mitglied der Deutschen Gesellschaft für Pathologie ist am 15. Oktober 2009 im Alter von 89 Jahren an einer Herzinsuffizienz verstorben.

Prof. Katsuo Ogawa wurde am 24. Januar 1920 in Takamatsu geboren. Er wurde im Jahre 1945 an der Medizinschen Hochschule zu Niigata, jetzt Medizinische Fakultät der Universität Niigata, graduiert. Im Jahre 1948 hat er bei Prof. Dr. Yukio Hamazaki, dem damaligen Direktor des II. Pathologischen Institutes Okayama (der Vorgänger von Prof. Katsuo Ogawa), mit den Forschungen über Pathologie begonnen.

Als Auslandsforscher des Japanischen Kulturministeriums hat Prof. Dr. Katsuo Ogawa im Jahre 1960 am Pathologischen Institut der Universität Hamburg (unter der Leitung des damaligen Direktors Prof. Dr. C. Krauspe) über die ultramikroskopische Morphologie der HodgkinKrankheit und über Obduktionen gear- beitet. Er war der erste japanische Pathologe, der sich die Befähigung zur Obduktion in Deutschland erworben hat. Auch anschließend stand Prof. Katsuo Ogawa in einem freundschaftlichen Verhältnis zur Deutschen Gesellschaft für Pathologie, weshalb ihn die Gesellschaft im Jahre 1990 als korrespondierendes japanisches Mitglied vorgeschlagen hat.

Im Jahre 1962 wurde Prof. Katsuo Ogawa Direktor des II. Pathologischen Institutes (jetzt Pathologisches Institut für Onkologie). Danach hat er bis zum Ruhestand im Jahre 1985 viel zu Ausbildung, Forschung und Diagnostik auf dem Gebiet der Pathologie beigetragen.

Nach der Rückkehr aus Deutschland hat Prof. Dr. Katsuo Ogawa seine Forschung auf die Krebsentstehung infolge der Adenovirusinfektion konzentriert. Der Erfolg wurde im Jahre 1979 unter dem Titel „Die von Adenovirus Typ 12 induzierte Geschwulst, besonders über den Versuch der experimentellen Gehirngeschwulst" speziell für die Tagung der Japanischen Gesellschaft für Pathologie vorgetragen.

Prof. Katsuo Ogawa war der Vizepräsident der 66. Tagung der Japanischen Gesellschaft für Pathologie, und er hat danach die 26. Tagung der Japanischen Gesellschaft für Neuropathologie als Präsident in Okayama ausgerichtet.

Prof. Katsuo Ogawa war stets freundlich und zu allen Kollegen auch zu Studenten immer vorbildlich im Verhalten. Weil er außerdem ein sehr vertrauenswürdiger Mann war, haben viele Kollegen bei ihm am II. Pathologischen Institut gearbeitet. Sie sind noch jetzt am $\mathrm{Pa}$ - thologischen Institut für Onkologie, an einigen Kliniken und vielen Krankenhäusern tätig.

Es gibt bei uns ein Motto aus dieser Zeit von Prof. Yukio Hamazaki und Prof. Katsuo Ogawa. Es lautet: „Weil die diagnostische (menschliche) und experimentelle Pathologie die beiden Räder eines Wagens sind, muss man sich um die beiden stets bemühen. " Prof. Katsuo Ogawa selbst hat sich immer darum daran gehalten und die Kollegen des Pathologischen Institutes dazu geführt.

Hiermit möchte ich Prof. Dr. Katsuo Ogawa für seine erfolgreiche Führung danken und zuletzt für seinen Seelenfrieden beten.

\section{Tadashi Yoshino, Okayama, Japan}

\section{Korrespondenzadresse \\ Prof. Dr. T. Yoshino \\ Department of Pathology, \\ Graduate School of Medicine, Dentistry and \\ Pharmaceutical Sciences, \\ Okayama University \\ 2-5-1 Shikata-cho, 700-8558 Okayama \\ Japan \\ yoshino@md.okayama-u.ac.jp}

Interessenkonflikt. Der korrespondierende Autor gibt an, dass kein Interessenkonflikt besteht.

Deutsche Obersetzung des Nachrufs, der in der Zeitschrift der Japanischen Gesellschaft für Pathologie veröffentlicht wurde. 